\title{
Bayesian Inference for Localization in Cellular Networks
}

\author{
Hui Zang \\ Sprint, USA
}

\author{
Francois Baccelli \\ ENS, France
}

Jean Bolot
Sprint, USA

\begin{abstract}
In this paper, we present a general technique based on Bayesian inference to locate mobiles in cellular networks. We study the problem of localizing users in a cellular network for calls with information regarding only one base station and hence triangulation or trilateration cannot be performed. In our call data records, this happens more than $50 \%$ of time. We show how to localize mobiles based on our knowledge of the network layout and how to incorporate additional information such as round-trip-time and signal to noise and interference ratio (SINR) measurements. We study important parameters used in this Bayesian method through mining call data records and matching GPS records and obtain their distribution or typical values. We validate our localization technique in a commercial network with a few thousand emergency calls. The results show that the Bayesian method can reduce the localization error by $20 \%$ compared to a blind approach and the accuracy of localization can be further improved by refining the a priori user distribution in the Bayesian technique.
\end{abstract}

\section{INTRODUCTION}

Mobile phones have become a fundamental component of modern lives and economies, and have become ubiquitous, reaching an estimated 4.1 billion by end of 2008 - over half of the planet's population - with several countries having penetration rates much higher than $100 \%$ [1]. As a result, recent research is looking at mobile phones as a powerful and exciting new tool to track and analyze human behavior, in particular human social interactions and activity patterns [2], [3].

Understanding human mobility patterns is of major importance in a number of areas, including of course cellular network design and engineering, but also urban planning, transportation geography, (mobile) advertising, crowd and event management, or epidemics monitoring and control. Until relatively recently, few tools and little large scale data were available to monitor the spatial dynamics of large populations of users. (For example, refer to [4] for a study of human mobility and travel patterns by studying the circulation of bank notes in the United States). The call data records (CDRs) collected by wireless operators for billing and troubleshooting purposes now provide one such source of data, and they make it possible to study human mobility patterns of populations at previously impossible-toachieve scales. The main challenge with CDRs has been availability. It remains limited, in particular because of privacy concerns and corresponding challenges with anonymization [5], but still availability has become greater in the past couple of years. We anticipate that there will be more work on CDRs and human mobility modeling can greatly benefit from the increasing availability of CDRs.

Our work is motivated by the need to convert large sets of CDRs to location records. CDRs contain only coarsegrained location information about the mobile user as cell and sector IDs and round-trip-time (RTT) and signal-to-noiseand-interference ratio (SINR) measurements. We would like to obtain more fine-grained location information. Although there has been a lot of work on localizing mobiles in a cellular network, most of them are based on the operation mode in which a mobile could see a few surrounding base stations [6], [7], [8], including the ones with very weak signals. However, only base stations with strong enough signals can carry a mobile's communication and only the base stations that were actually carrying the mobiles' communication were logged into CDRs. Although during handoffs, would two or more base stations carry a call simultaneously, in all other cases, 50\% of our CDRs, the mobile is served by only one base station. In order to convert CDRs to location records, we need to deal with the majority cases where there is only one base station recorded. Clearly popular localization techniques such as TOA, AOA [6], [9], or signal-strength fingerprinting [10] are not applicable because they require two or more distinct base stations. Therefore, we need to solve the problem of localization for these single-legged calls.

We develop a Bayesian-based method to localize users in cellular network with only one call-leg information. We consider information such as the distance to the base station, location of neighboring base stations, and levels of interference and/or noise. We demonstrate the benefits of our technique with CDRs of E911 calls with matching GPS coordinates. We can improve the localization accuracy by $20 \%$ comparing to a blind approach in which a location is randomly chosen along an arc of the sector and the arc is determined based on distribution of RTT measurements.

Although our work is motivated by offline call data processing, the technique developed in this work also benefits locationbased mobile applications which do not have direct access to information from several cell/sectors to perform localization using the alternative methods (TOA, AOA, fingerprinting). Our approach is very general and applicable not just to cellular networks, but to other wireless networks in particular wireless LANs.

The rest of the paper is organized as follows. Section II provides background information about the network and the data set under study and review related work. We develop the Bayesian-based method in steps in Section III] Section IV] presents measurement results that aid us in selecting parameters for the Bayesian method. The method is evaluated in Section $\mathrm{V}$ and Section VI concludes the paper.

\section{BACKGROUND}

\section{A. Network Information}

We consider a commercial CDMA2000 network which carries voice, data and SMS traffic. We obtain a network map with locations of base stations as (latitude, longitude) pairs. In the network, all base stations are equipped with directional antennas and each cell has two or three sectors. We know the azimuth (direction) of each antenna, which corresponds to the 
center axis of each sector, together with the beam-width of each antenna, which determines the angle coverage of the sector. In this paper, we consider a metropolitan area of about 7000 square miles or 18000 square kilometers. This area is covered by four CDMA switches and about one thousand base stations. The majority of the cells are located in suburban areas with the exception of a minor fraction belonging to an urban area.

In localization, a key factor that determines the accuracy is the separation distance between anchor points, i.e., base stations in cellular networks. In cellular networks, the separation distance varies a lot from urban to suburban areas, and from freeway cells to residential cells. For the network under study, the average separation distance is around 2 kilometers.

\section{B. Data: GPS Records and Call Data Records}

The first data set we use consists of GPS locations of three months of E911 calls originated from the network. The GPS coordinates serve as our ground truth. This set also contains the information about the cell and sector that carry each call.

Another data set is referred to as "Call Data Records" (CDRs). This is a separate data set and we match the E911 calls to records in this set. Then we perform localization with information from CDRs and calculate the error of the results against the GPS coordinates.

A call record consists of over 100 fields with information related to both the mobile device and the cellular network. All calls on a network, including voice, data, and SMS calls, are logged. In this study, we are particularly interested in the following information in the call records: 1) start and end time of call; 2) starting and ending base stations and sectors; 3) round-trip-time (RTT) between the mobile device and the BS; and 4) Signal to Noise and Interference Ratio (SINR) of the base station measured by the mobile device.

\section{Related Work}

There has been a large body of work in localizing mobile phones without GPS support, mainly driven by location based services [11], including the E911 service [12]. Because cell phones may not be equipped with GPS or be at places with poor GPS reception, network-assisted localization is needed.

Most localization techniques rely on time-of-arrival (TOA) or angle-of-arrival (AOA) measurement from nearby base stations [6], [9]. TOA provides the delay information between the mobile and the base station and therefore with TOA, the mobile will be on a circle centered at the base station, with the radius of the circle given by TOA. With accurate TOA measurement from one base station, [8] demonstrate that the error in localizing mobiles in a UMTS network is above $400 \mathrm{~m}$. Better localization accuracy can be achieved by using TOA from three surrounding base stations, which is called trilateration, AOA estimates the angle at which the base station's signal comes in. With AOA from two neighboring base stations, a mobile's location can be easily identified. This process is referred to as triangulation. When the mobile does not have a line-ofsight (LOS) signal from the base station, both TOA and AOA will be inaccurate. Research has shown that the accuracy of localization can be improved by overcoming the inaccuracy in TOA and AOA measurements [13] and by using both TOA and AOA from neighboring base stations [13], [14]. Localization is also feasible when no AOA or TOA information is available. In this case, the overlapping coverage areas of three or more neighboring base stations are considered [15]. Besides TOA and
AOA, signal-strength fingerprints can also be used to localize a mobile. In [10], signal strength from six or more cells are used to form a fingerprint and the fingerprint based localization gives median accuracy of 5 meters in an indoor environment. In another study [16], fingerprint based techniques are shown to outperform a centroid algorithm. For all fingerprint based methods, a training process is required to collect the fingerprint of locations and the quality of the training set affects the accuracy of localization.

Bayesian inference, based on conditional probability, is a powerful statistical tool for dealing with measurement uncertainty and combining multiple sources of information [17]. Refer to [17] for a survey of various implementations of Bayesian filters and how they are applied to location estimation with ultrasound and infrared technologies. Authors in [18] propose a localization technique using Bayesian inference with signal strength measurement in an indoor WiFi environment. In this paper, we develop a Bayesian-based localization technique using information from only one cell/sector.

Much research has been done for localization in WiFi networks. Although underlying techniques are similar, the outcome of these techniques differ for WiFi networks and for cellular networks in several aspects including network coverage, infrastructure stability, and separation distance between network nodes [10]. Reference [19] presents a localization platform using both fingerprints from GSM and WiFi.

\section{BAYESIAN INFERENCE}

In the presence of only coarse grained information of a call such as associated base station $X_{0}$, and without $a$ priori information on the user location, it makes sense to assume that the user is uniformly located in the Voronoi cell of $X_{0}$ (the set of points which are closer to base station $X_{0}$ than to any other base station [20]). The CDRs include sector information as well. Cells with omni-directional antennas belong to the above case where no sector information is known. For cells with directional antennas, sectors are characterized by the direction (azimuth) of the antennas and their horizontal beamwidth. Therefore, sector gives more information about the user's location. Suppose the user is in sector $s_{0}$. The uniform measure on the Voronoi cell should be replaced by the uniform measure of the sector. Next in CDRs, we have the round-trip-time (RTT) measurement between the base station and the mobile, and therefore distance information. This RTT is estimated with an inherent error. Given that this distance is $r$ with an error range $\pm \delta$, we now should replace the uniform measure of the entire sector by the uniform measure of the part of the ring of average radius $r$, width $2 \delta$, center $X_{0}$, and belonging to the sector $s_{0}$. In the remaining of the section, we develop a method to estimate user locations based on the aforementioned information.

As a starting point, we assume we know only the associated base station $\left(X_{0}\right)$. We also know the network layout, i.e., the mapping of base station IDs to geographical locations.

\section{A. With Information of Associated Base Station}

More generally, consider a user located at $x$ in the neighborhood of a set of base stations $X_{0}, X_{1}, \ldots, X_{K}$. The question considered here is that of the evaluation of the probability that this user is located at $x$ given it is attached to the base station $X_{0}$. Because radio conditions may vary considerably due to scattering, particularly so in urban areas, the resulting fading phenomenon implies that users may be attached to another 
base station than the closest, which is a well documented observation. Thus we need to consider a model which takes fading into account. In what follows, we will assume to know

- Some finite neighborhood $\Delta$ of $x$ (more precisely, $\Delta$ is some convex subset of the plane that contains the location $x)$;

- The set $\left\{X_{0}, X_{1}, \ldots, X_{K}\right\}$ of all base stations in the domain $\Delta$.

Here are a few simple examples:

1) $\Delta$ is the disc of center $X_{0}$ and radius $r$ where $r$ is a pre-defined value; $\left\{X_{1}, \ldots, X_{K}\right\}$ are the base stations in this disc.

2) $\left\{X_{1}, \ldots, X_{K}\right\}$ is the set of Delauney neighbors [20] of $X_{0}$ w.r.t. the collection of points $\left\{X_{0}, X_{1}, \ldots, X_{K}\right\} \cup$ $\{\mathcal{X}\}$, where $\{\mathcal{X}\}$ denotes the other base stations of the network.

We assume that the mean attenuation for power at distance $d$ is of the form $\alpha d^{\beta}$, with $\beta>2$ and $\alpha$ being $(4 \pi)^{\beta}$. We also assume a Rayleigh fading from any base station to each given point $x$ of the plane. It is well known that the effect of Rayleigh fading on power attenuation is equivalent to the multiplication by an exponential random variable with mean 1 [21]. The method can be extended to other fading cases too. In later sections of the paper, we also consider a fading of which the power attenuation follows a Gamma distribution.

We introduce the following notation:

- Let $p_{i}, 0 \leq i \leq K$ denote the mean total power used on the downlink of station $X_{i}$.

- Let $F_{i}, 0 \leq i \leq K$ denote an exponential random variable with mean 1 , which represents the fading from $X_{i}$ to $x$.

- Let $I_{\mathcal{X}}(x)$ denote the power of the interference created at point $x$ by all the base stations of $\{\mathcal{X}\}$.

- Let $N(x)$ denote the thermal noise power at $x$.

- Let $L_{i}(x), 0 \leq i \leq K$ denote the path loss from base station $X_{i}$ to location $x$ :

$$
L_{i}(x)=\frac{1}{\alpha\left\|x-X_{i}\right\|^{\beta}}, \quad \beta>2, \alpha=(4 \pi)^{\beta} .
$$

The random variables $F_{0}, F_{1}, \ldots, F_{K}$ are assumed independent. The locations of the base stations $X_{0}, X_{1}, \ldots, X_{K}$ are known. There are natural ways of estimating $I_{\mathcal{X}}(x)$ and $N(x)$. For the time being, we only assume that these variables are independent of the fading variables and that the Laplace transforms of these two variables, respectively denoted by $\Psi_{I_{\mathcal{X}}(x)}(s)$ and $\Psi_{N(x)}(s)$, are known.

Our general technique proceeds in two steps. Step 1 is to compute the probability $\pi_{X_{0}}(x)$ for base station $X_{0}$ to "cover' 1 a user located at $x$. Step 2 is to use Bayes' formula to compute $\phi(x)$, the density of the user location, conditional on the fact that the user is attached to station $X_{0}$. We next describe these steps in details.

1) Step 1: We compute the probability $\pi_{X_{0}}(x)$ for base station $X_{0}$ to "cover" a user located at $x$, assuming that coverage happens when the signal to interference plus noise ratio (SINR) at the user is more than some threshold $\gamma$.

$$
\begin{aligned}
\pi_{X 0}(x)= & P\left(p_{0} F_{0} L_{0}(x) \geq\right. \\
& \left.\gamma\left(\sum_{i=1}^{K} p_{i} F_{i} L_{i}(x)+I_{\mathcal{X}}(x)+N(x)\right)\right)
\end{aligned}
$$

\footnotetext{
${ }^{1}$ In the paper, we use the following phrases interchangeably: "to cover", "to carry the communication of", and "to be in the active set of".
}

Since $F_{0}$ is exponential, and using the independence assumption, we can rewrite the last expression as

$$
E\left(e^{-\frac{\gamma}{p_{0} L_{0}(x)}\left(\sum_{i=1}^{K} P_{i} F_{i} L_{i}(x)+I_{\mathcal{X}}(x)+N(x)\right)}\right)
$$

With integration over $F_{i}, 1 \leq i \leq K$, we obtain

$$
\begin{aligned}
\pi_{X_{0}}(x)= & \prod_{i=1}^{K} \frac{1}{1+\gamma \frac{p_{i} L_{i}(x)}{p_{0} L_{0}(x)}} \cdot \Psi_{I_{\mathcal{X}}(x)}\left(\frac{\gamma}{p_{0} L_{0}(x)}\right) \\
& \cdot \Psi_{N(x)}\left(\frac{\gamma}{p_{0} L_{0}(x)}\right)
\end{aligned}
$$

2) Step 2: We can now use Bayes' formula to compute the density of the user location conditional on the fact that the user is attached to station $X_{0}$ as

$$
\phi(x)=\frac{\pi_{X_{0}}(x) f(x)}{\int \pi_{X_{0}}(u) f(u) d u},
$$

where $f($.$) is the a priori density of the user location.$

Consider a few special cases. In the case when there is no $a$ priori knowledge on the location of the user, one should take $f$ uniform on the domain $\Delta$ (as discussed earlier in this section). The density $\phi(x)$ is then computed with respect to the Lebesgue measure on the plane and is

$$
\phi(x)=\frac{\pi_{X_{0}}(x)}{\int_{\Delta} \pi_{X_{0}}(u) d u} .
$$

In the case when RTT is known, i.e. the user is known to be located at distance $r$ from $X_{0}$, then one should take $f$ uniform on the circle $C$ of radius $r$ and center $X_{0}$. The density $\phi(x)$ is then computed with respect to the Lebesgue measure on $C$ and is

$$
\phi(\theta)=\frac{\pi_{X_{0}}(r, \theta)}{\int_{0}^{2 \pi} \pi_{X_{0}}(r, \alpha) d \alpha} .
$$

To evaluate $\Psi_{N(x)}$, we can use classical models for thermal noise, the simplest one being the constant case. $\Psi_{I_{\mathcal{X}}(x)}$ can be estimated if the density of the base stations is known [22]. Details are omitted for the sake of space. We will shown in Section V that interference from other non-neighboring base stations can be neglected in some cases.

3) Numerical Examples: The method can be illustrated by an example. Suppose we know that the user is associated with base station $X_{0}$ located at $(0,0)$ and the domain $\Delta$ is the disc of radius 3 and center $X_{0}$; the set of base stations in $\Delta$ is $X_{1}=(1,1), X_{2}=(1,-1)$ and $X_{3}=(-2,-1)$, as illustrated in Fig. 1(a).

a) 2-D densities: Figure 1(b) shows the 2-D density $\phi$ (up to a multiplicative constant). The thermal noise is constant and equal to $1 ; \gamma=1$, all mean powers are equal to $1, \beta=4$ and $\lambda=1$.

b) 1-D densities: We compute the density for the case in which the user is at distance 1 from the attached base station $X_{0}$. Figure $1(\mathrm{c})$ shows the density with $\gamma=0.4$ in polar coordinates. Comparing it with Fig. 1(a), we can see that when there is no a priori information on the user location (more than distance to the base station), the chance to find the user is lower in the regions that are closer to neighboring base stations. Figures 2(a) and 2(b) show the 1-D density in angular coordinates with $\gamma=1$ and $\gamma=0.2$, respectively. 


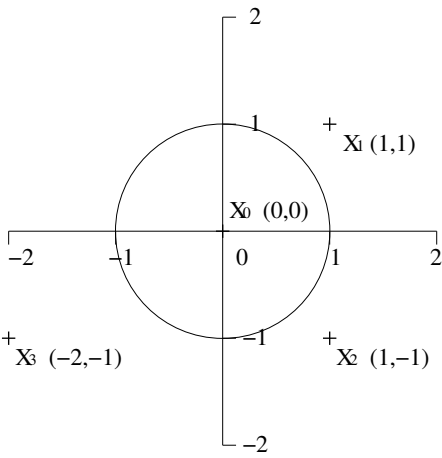

(a)

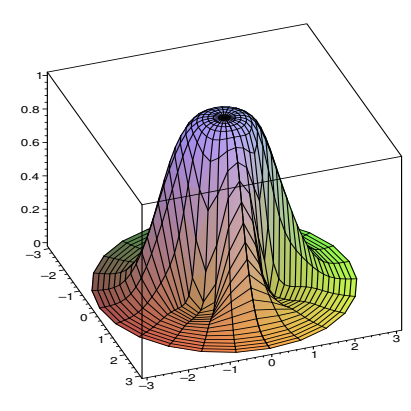

(b)

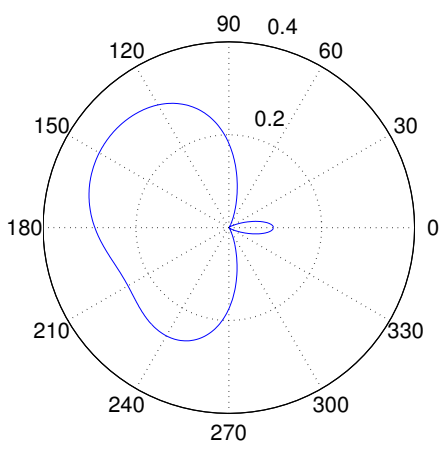

(c)

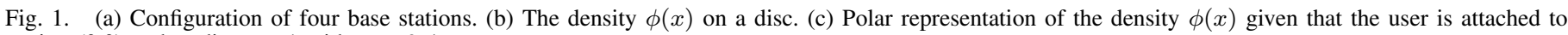
station $(0,0)$ and at distance 1 with $\gamma=0.4$.

\section{B. With Sector Information}

In sectorized cells, in addition to the attenuation introduced by the distance between the base station and the mobile, there will be an additional attenuation associated with the angle from the azimuth, i.e., the center axis of the sector. In other words, at the same distance to the base station, a user located farther off the azimuth receives on average less power than a user located right on or near the azimuth. According to [23], a distanceand-angle combined path-loss function for a directional antenna with azimuth $\theta_{0}$, located at $X_{0}$, for a point $x, x, X_{0} \in \mathcal{R}^{2}$, can be written as:

$$
L\left(x, X_{0}, \theta_{0}\right)=l\left(\left\|x-X_{0}\right\|\right) G\left(\arg \left(x-X_{0}\right)-\theta_{0}\right),
$$

where $l$ is the omni-directional attenuation function shown in Eqn. (1), and $G$ is the normalized radiation pattern. $\left\|x-X_{0}\right\|$ and $\arg \left(x-X_{0}\right)$ represent the Euclidean norm and the argument of the vector from point $X_{0}$ to point $x$, respectively.

For directional antennas, the parameter beam-width is defined as follows. At an angle equal to half of the beam-width, the power is half of what the power is at the azimuth. For example, for an antenna with beam-width of $2 \pi / 3$, if the power at location $a$, which is of distance 1 from the antenna, along the azimuth, is 1 , then the power at location $b$, which is also of distance 1 from the antenna, but at $\pi / 3$ angle, will be 0.5 .

The radio pattern function $G$ can be formulated as:

$$
G(\theta)=\left|\frac{\sin (\omega \theta)}{\omega \theta}\right|, \quad|\theta| \leq \pi,
$$

where $\omega$ is chosen such that $G(\theta)=1 / 2$ when $\theta$ is half beamwidth. Reference [23] gives $\omega=1.81$ for antenna with beamwidth $2 \pi / 3$. Therefore, for an antenna with beam-width $\theta_{0}$, $\omega=1.81 \times(2 \pi / 3) / \theta_{0}$.

We can easily extend our localization method to include sector information by letting $X_{0}$ represent the sector which the mobile is associated with, and $X_{i}, 1 \leq i \leq K$ represent a member in the neighbor sector set and replacing the omnidirectional path-loss function Eqn. (1) with sectorized path-loss function Eqn. (7).

\section{More SINR information}

CDR records SINR information about each call leg with certain accuracy. Therefore, instead of using a fixed SINR threshold $\gamma$, we can take advantage of this knowledge and assume the actual SINR falls on the range $\hat{\gamma} \pm \epsilon$, where $\hat{\gamma}$ is

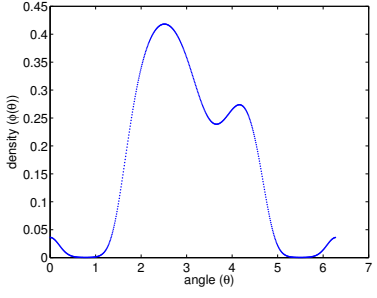

(a) $\gamma=1$

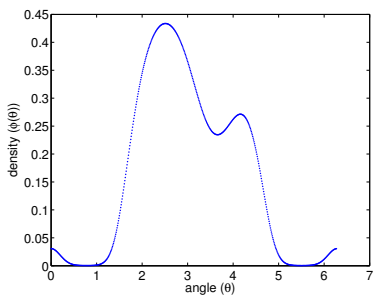

(c) $\hat{\gamma}=0.5 \pm 1 d B$

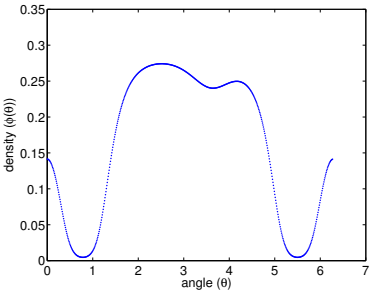

(b) $\gamma=0.2$

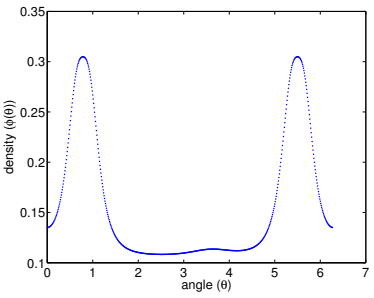

(d) $\hat{\gamma}=0.2 \pm 1 d B$
Fig. 2. The density $\phi(\theta)$ in angular coordinates, given that the user is attached to station $(0,0)$ and is at distance 1 .

the reported SINR and $\epsilon$ is a small value. Let $\pi_{\gamma}(\theta)$ denote the probability of a mobile having a SINR greater than $\gamma$ given that the mobile is at direction $\theta$, which is calculated by Eqn. (3), then the probability that a mobile has a SINR value in range $(\hat{\gamma}-\epsilon, \hat{\gamma}+\epsilon)$ given that the mobile is at direction $\theta$, can be calculated as:

$$
\pi(\theta)=\pi_{\hat{\gamma}-\epsilon}(\theta)-\pi_{\hat{\gamma}+\epsilon}(\theta) .
$$

Followed with step 2, we will obtain the density function $\phi(\theta)$.

In the example network shown previously, we can obtain the density function with knowledge of the SINR range. Figure 2 shows that a measured $\hat{\gamma}=0.5$ gives the similar distribution as $\gamma=1$ (Fig. 2(c)) while the distribution based on $\hat{\gamma}=0.2$ is dramatically different (Fig. 2(d)).

\section{D. "Hidden" SINR information of neighbors}

CDR records all base station sectors that are in the mobile's active set at the time of the call. If the SINR from a sector is above a certain threshold, the sector is placed in the so-called "active set". The fact that only one sector is recorded in the 
CDR tells us that all other neighboring base station sectors have lower SINR and do not make to the active set. Therefore their SINRs must be lower than a threshold. These two thresholds may or may not be the same ${ }^{2}$ We can formulate this additional information.

Consider now the case where the mobile at $x$ is covered by base station $X_{0}$, which happens when the SINR of $X_{0}$ is more than $\gamma$, and that the neighboring base stations $X_{i}, i=1, \ldots, K$ are not in the active set of the mobile, which happens when the SINR of $X_{i}$ is less than $\nu$. Let $C(x)$ denote the sum of the thermal noise and interference from non-neighboring base stations at $x$, the probability of this event, $\pi_{X_{0}, \bar{X}_{1}, \ldots, \bar{X}_{K}}(x)$, is

$$
\begin{aligned}
& P\left(p_{0} F_{0} L_{0}(x)>\gamma\left(\sum_{i=1}^{K} p_{i} F_{i} L_{i}(x)+C(x)\right),\right. \\
& \left.\cap_{j=1}^{K} p_{j} F_{j} L_{j}(x)<\nu\left(\sum_{i=0, i \neq j}^{K} p_{i} F_{i} L_{i}(x)+C(x)\right)\right),
\end{aligned}
$$

where $L_{i}(x)$ denote the path loss factor at location $x$ from base station $X_{i}$. Therefore,

$$
\begin{aligned}
& P\left(P_{0} F_{0} L_{0}(x)>\max \left(\gamma\left(\sum_{i=1}^{K} p_{i} F_{i} L_{i}(x)+C(x)\right),\right.\right. \\
& \left.\left.\vee_{j=1}^{K}\left(\frac{p_{j} F_{j} L_{j}(x)}{\nu}-\sum_{i=1, i \neq j}^{K} p_{i} F_{i} L_{i}(x)-C(x)\right)\right)\right) .
\end{aligned}
$$

Since the $F_{i}$ 's are exponential and independent, we deduce from the last expression that

$$
\begin{gathered}
\pi_{X_{0}, \bar{X}_{1}, \ldots, \bar{X}_{K}}(x)=\int_{a>0} \int_{t_{1}>0} \cdots \int_{t_{K}>0} \\
e^{-\frac{g\left(x, t_{1}, \ldots, t_{K}, a\right)}{p_{0} L_{0}(x)}} e^{-t_{1}} \cdots e^{-t_{K}} d t_{1} \ldots d t_{K} f_{x}(a) d a
\end{gathered}
$$

where $f_{x}$ denotes the density of $C(x)$ and

$$
\begin{gathered}
g\left(x, t_{1}, \ldots, t_{K}, a\right)=\max \left(\gamma\left(\sum_{i=1}^{K} p_{i} t_{i} L_{i}(x)+a\right)\right. \\
\left.\vee_{j=1}^{K}\left(\frac{p_{j} t_{j} L_{j}(x)}{\nu}-\sum_{i=1, i \neq j}^{K} p_{i} t_{i} L_{i}(x)-a\right)\right)
\end{gathered}
$$

The Bayesian inference method can then be used in the same

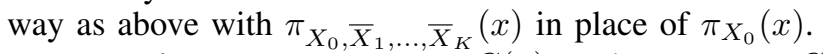

In practice, we can assume $C(x)$ to be constant $C$ and Eqn. (10) can be simplified for one integration dimension.

The equation above is written for the omni-directional antenna model where $X_{i}$ represents base station $i$. They can also apply to the sectorial-antenna model where the neighbor set consists of all sectors from neighboring base stations and $X_{i}$ denote a particular sector in this set. The only difference is in the calculation of the path loss factor $L_{i}(x)$. Note that we cannot obtain a closed-form solution and hence have to rely on numerical evaluation. To keep the complexity low enough in the sectorial case, we can limit the neighboring sectors to a small number.

\footnotetext{
${ }^{2}$ In network operations, an active set member will be removed from the active set if its SINR is lower than a certain threshold and this threshold may be different from the threshold of adding a member to the active set.
}

\section{E. Inaccurate RTT Information}

Consider now the case when the only a priori knowledge is the RTT from the base station to the mobile and this RTT is error-prone. Assume that the estimated distance between the base station and the mobile is $r$, and the actual distance of the mobile to the base station has distribution $f_{r}$ (for instance the truncation on the positive real line of a Gaussian distribution with mean $r$ ). Then, given $r$, our a priori information on this distance is $f_{r}$. In the omni-directional antenna case, the density $\phi$ in polar coordinates is then

$$
\begin{gathered}
\phi(u, \theta)=\frac{\pi_{X_{0}}(u, \theta) f_{r}(u)}{\int_{v=0}^{\infty} \int_{\alpha=0}^{2 \pi} \pi_{X_{0}}(v, \alpha) f_{r}(v) d v d \alpha} . \\
\text { IV. ESTIMATING PARAMETERS }
\end{gathered}
$$

\section{A. Power fading distribution}

The heart of our computation relies on the assumption that the fading is Rayleigh, for which it has been proved that the effect on power attenuation can be modeled as an exponentiallydistributed random variable [21]. We validate this assumption by measuring the received power at a particular location by a device. We collect time series of pilot power using Agilent W1314A Multi-band Wireless Measurement Receiver [24]. The device records the power levels of all perceived pilots around every 0.3 seconds. We collect multi-day traces at three different locations and focus on the strongest pilot at each location. For each location, we partition the traces into day-long pieces and study each piece separately. Locations $A$ and $C$ are suburban locations and Location $B$ is inside a big city. We take one day each from Locations $A$ and $B$ and two days which are five days apart from Location $C$. For each trace, we calculate the mean and then divide each sample by this value, based on the assumption that the fading factor has mean 1 . The probability density function (PDF) of the resulted ratio is plotted in Fig. 3 . We fit Gamma distribution to each data set and the PDF of the corresponding Gamma distribution is also plotted in each figure. The legends in the figures gives the two parameters $a$ and $b$ for the Gamma distribution, with $a$ for the shape and $b$ for the scale. We know that Gamma distribution becomes Exponential distribution when $a=1$. We notice that the two suburban locations ( $A$ and $C$ ) has $a$ between 1 and 2 while the urban location $B$ has $a$ much larger than one. The measurement results indicate that Rayleigh fading can be used to approximate the fading effect in suburban areas while it becomes less accurate in urban areas. This discovery suggests the need for an extension of our method to other types of fading. We consider a simple Gamma distribution with $a=2$ in the next section and extensions with more generalized fading cases is a topic for future research.

\section{B. SINR}

The SINR values recorded in CDR are of $0.5 d B$ accuracy. In order to obtain a proper SINR threshold value for localization, we first classify SINR values into three categories:

- SINRs reported in CDRs with only one call leg;

- SINRs reported on the primary leg (usually the strongest leg) when the call has multiple legs.

- SINRs reported on the non-primary legs when the call has multiple legs.

We plot the complementary cumulative distribution function (CCDF) of the three SINR groups in Fig. 4. We observe from Fig. 4 (a) that $80 \%$ of the single-legged calls had a SINR value 


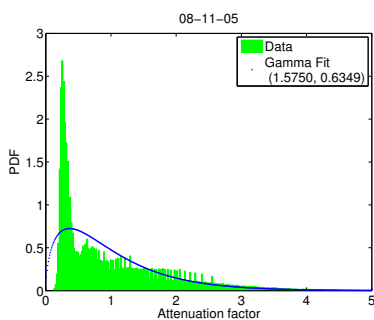

(a) Location $\mathrm{A}$

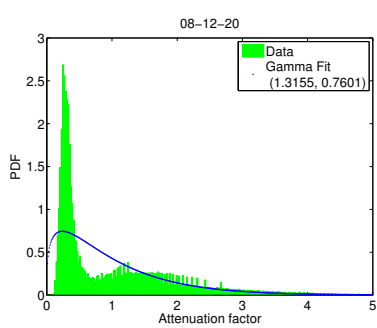

(c) Location $\mathrm{C}$

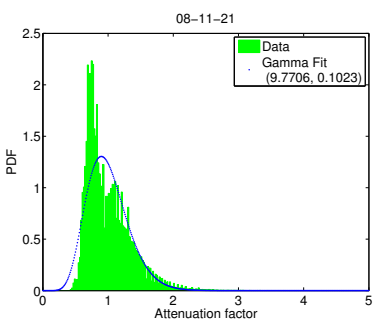

(b) Location B

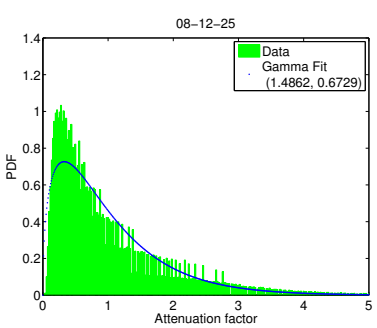

(d) Location $\mathrm{C}$
Fig. 3. PDF of power fading factor measured at three locations.

greater than $-7 d B$, or 0.2 , and $50 \%$ had a SINR value greater than $-5 d B$, or 0.3 . As a comparison, the SINRs on the primary leg in multi-legged calls are lower, and only $20 \%$ had a SINR value greater than $-5 d B$. SINRs on the non-primary legs in multi-legged calls are even lower, $60 \%$ are greater than $-12 d B$, or $40 \%$ are lower than $-12 d B$, or 0.06 .

We are also interested in how stable the SINR is at one location. Therefore we carried out an active experiment which involved two phones, placed side by side at a location, receiving calls every five minutes. In each set of the experiment, which we refer to as Test 1 or Test 2, we sent 300 SMSes to each phone in three batches, with batches separated by a few hours, and the two tests are seven-days apart. Each SMS call was logged in the CDR database. The PDF of the SINR values are shown in Fig. 5 We can see that in both tests, the SINR values are more spread out for Mobile 1 than for Mobile 2. However, the mean SINRs are fairly stable, i.e., between 0.23 and 0.29 at the given location. This fact indicates that if we have repeated measurements at a location, the mean SINR can give more information regardless of the time of measurement or the type of the phone.

\section{RTT}

In this subsection, we study the round-trip-time reported by mobiles and compare it with the actual distance calculated by the GPS coordinates. Note we need to divide the RTT by two to obtain the one-way delay. Figure 6 summarizes the error in reported delay in the data set. Error is computed as the measured delay subtracted by the actual distance. Note that the error can be fitted with a normal distribution with mean and standard deviation both around $0.3 \mathrm{~km}$. We also examine the delays between the two mobiles and the associated base station in the active experiment (Section IV-B). The distance between the location of the experiment and the associated base station, calculated by their GPS coordinates, is $382 \mathrm{~m}$, while the average of reported delay is $745 \mathrm{~m}$ for mobile 1 and $760 \mathrm{~m}$ for mobile 2. Therefore there is on average $363 \mathrm{~m}$ error in mobile 1 's reported delay. and $378 \mathrm{~m}$ in mobile 2's reported delay. This

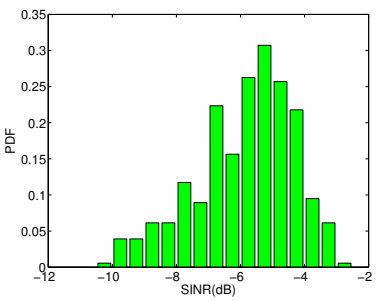

(a) Mobile 1, Test $1(0.291)$

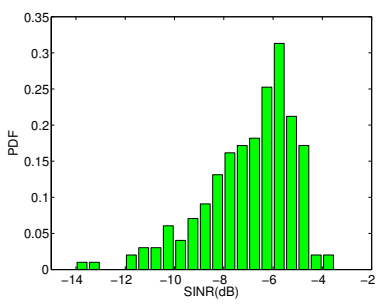

(c) Mobile 2, Test $1(0.230)$

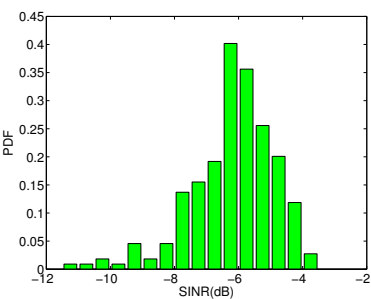

(b) Mobile 1, Test 2 (0.266)

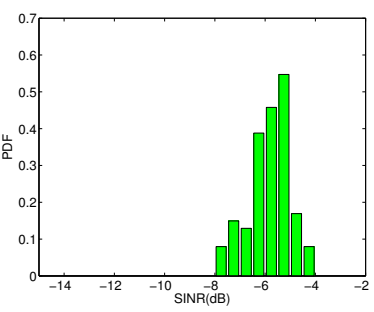

(d) Mobile 2, Test 2 (0.281)
Fig. 5. PDF of SINR from two mobiles at the same location. Values in parentheses indicate the mean.

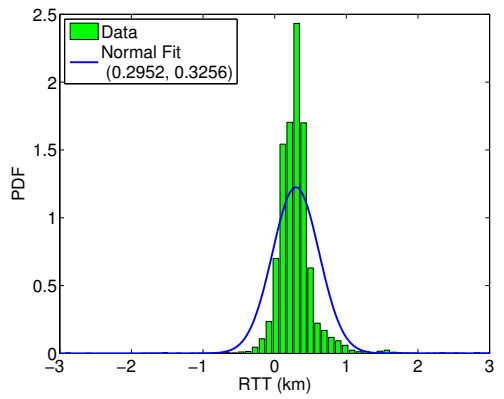

Fig. 6. PDF of delay measurement error for single-legged calls.

error, if not treated carefully, will introduce significant error in localization.

\section{Putting It Together: Numerical Evaluation}

Our evaluation is based on an E911 call database which records the GPS coordinates (latitude, longitude pairs) and the cell/sector IDs associated with the mobile for each E911 call. We match these records to CDRs and focus on the E911 calls with only one call-leg in the corresponding CDR. We then perform localization with the information provided by CDRs and calculate the localization error with the GPS coordinates. Strictly speaking, the GPS coordinates can contain errors as well. However, we can safely ignore this error because its magnitude is much lower than the accuracy of the localization algorithm. In what follows, we will see that the localization error is above a few hundred meters, whereas GPS has errors at a few meters up to tens of meters.

Recall that our data comes from four switch areas (Section III). For each switch area, there are two time periods during which the GPS data was collected and each period lasted three consecutive months. The two time periods are one year apart. The CDRs were collected correspondingly. Therefore, we have eight sets of E911 calls. We evaluated our localization method with one set in which there are about ten thousand E911 calls in the three month period and about five thousand calls with one call leg. The corresponding switch area consists of about 


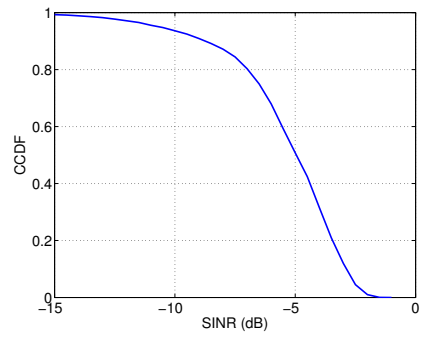

(a) One leg

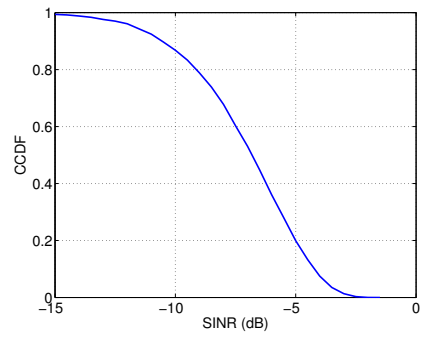

(b) Multiple legs: reference leg

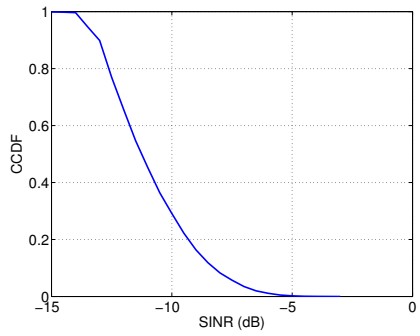

(c) Multiple legs: non-reference leg

Fig. 4. CCDF of reported SINR from a switch area.

250 cells and most of which are in suburbs. We repeated our evaluation on the other set of calls in this switch area and the results were similar. We also repeated our evaluation for other switch areas. The absolute localization error varies slightly with the separation distance for each switch area and we skip the details due to space limitation. Below we report the results from the five thousand single-legged calls in the selected data set.

Note that RTT measurement has an intrinsic error due to multi-path [7]. In the rest of the section, we first consider the simplified case in which the true RTT is known and we use the Bayesian method to estimate the angle. Then we consider the error distribution of the RTT measurement and compute the density function for $(r, \theta)$ pairs using Eqn. (12).

When knowing only the associated sector, we can assume that the user is uniformly distributed along the part of the ring that is of distance $r$ away at the center arc, within the angle range given by the beam width, and $r$ is the measured one-way delay, corrected by the mean RTT error, and the width of the ring is the standard deviation for the RTT error distribution. This will be our a priori distribution. As a baseline approach, we sample a uniformly-distributed random variable for the angle along the arc, and another random variable with Gaussian distribution for the distance from the base station. The resulted location would be the output.

The resulted mean error is $501 \mathrm{~m}$ and the root mean square error (RMSE) is $891 \mathrm{~m}$. If we know the actual RTT, we can reduce the mean error to $431 \mathrm{~m}$ and RMSE to $774 \mathrm{~m}$. If we have no knowledge of the RTT error and just use the measured RTT as input, the mean error would be $641 \mathrm{~m}$ and the RMSE $985 \mathrm{~m}$.

Next we perform the Bayesian-based inference method to locate mobiles on this ring segment. For the Bayesian method, we use the location that has the maximum density (or the maximum likelihood). We can lower the mean error to around $400 \mathrm{~m}$ (from $501 \mathrm{~m}$ for the baseline approach) without actual RTTs and to $355 \mathrm{~m}$ (from $431 \mathrm{~m}$ for the baseline approach) with actual RTTs. We describe our evaluation parameters and results in more detail in the rest of this section.

\section{A. Impact of $\gamma, \beta, K, N(x)$ and $I_{\mathcal{X}}(x)$}

To start, we choose $\beta=4$. We consider a constant thermal noise as $N(x)=5 f W$, or $5 \times 10^{-15} W$ [25]. The calculation is based on a temperature around $20^{\circ} \mathrm{C}$ and for the CDMA2000 bandwidth $1.25 \mathrm{MHz}$. The interference from sources other than the neighboring cells/sectors $I_{\mathcal{X}}(x)$ are assumed to be negligible, i.e., $I_{\mathcal{X}}(x)=0$. Recall from Section III that $\{\mathcal{X}\}$ denotes the base stations of the network not in the neighbor set and $I_{\mathcal{X}}(x)$ denote the power of the interference created at point $x$ by base stations in this set. Therefore, the combined power of noise and interference from non-neighboring set, $C$, is $5 f W$.

We first examine an implementation of Eqn. (3) with sector information where we consider the associated base station sector has $\operatorname{SINR}>\gamma$ and $\gamma=0.2$. The mean localization error is about $355 \mathrm{~m}$ and the RMSE is $681 \mathrm{~m}$. Compared to the baseline case when mean error is $431 \mathrm{~m}$ and RMSE $774 m$, the improvement in mean error is $17 \%$. We also repeated the inference with $\gamma=0.4$ and discovered that the mean error increases for less than $0.1 \%$.

When we change $\beta$ from 4 to 3 , the mean localization error is increased by $12 \%$. Therefore, $\beta=4$ represents the distancerelated attenuation exponent in this area.

We also repeated the inference with different values of $C=0.5,5,10,50 \mathrm{fW}$. Table I gives the associated localization errors.

TABLE I

PERFORMANCE WITH DIFFERENT NOISE VALUES.

\begin{tabular}{c|c|c|c|c}
\hline$C(f W)$ & 0.5 & 5 & 10 & 50 \\
\hline Mean Localization Error $(\mathrm{m})$ & 364.3 & 354.9 & 354.7 & 356.8 \\
\hline
\end{tabular}

The best $C$ values are around $5 f W$ and $10 f W$, which indicates $I_{\mathcal{X}}(x)=0$ or $I_{\mathcal{X}}(x)=5 f W$ for $N(x)=5 f W$. Therefore, the interference from non neighboring base stations is either negligible or at most of the same magnitude as the thermal noise. For the remaining evaluation, we used $C=10$.

In the results above, We considered three neighboring base stations in addition to the associated base station $X_{0}$ for any given point $x$, i.e., $K=3$. Note that the neighbor set changes with $x$, or $\theta$. When $\theta=\pi / 2$, the base stations to the north of $X_{0}$ are more likely to be included in the neighbor set and when $\theta=0$, the base station to the east are more likely to be considered. When we reduce $K$ to 2 , the resulted localization error is about the same. The reason is that in the switch area under study, the 3rd closest base station to a point $x$ besides the associated base station is usually at a distance that is more than $2 \mathrm{~km}$ to $x$ and the interference is negligible. Therefore, we can use $K=2$ in the future for such suburban cells. However, in a city where base stations are placed at a higher density, we may need $K>2$.

\section{B. More SINR information}

Since we do have the SINR information from the CDR, can we use this information to refine our estimation? To answer this question, we take the reported SINR $\hat{\gamma}$, and assume the "actual" SINR is within the range $\hat{\gamma} \pm \epsilon$. We tested with $\epsilon=1,2,3 d B$ 


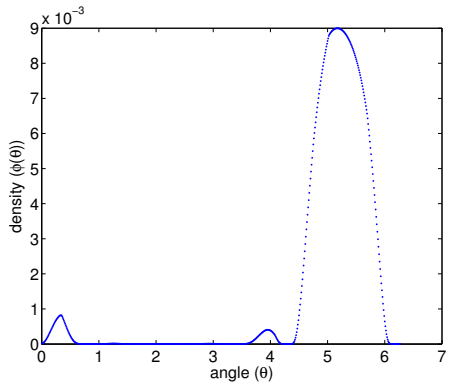

(a) No neighbor info., Exp. dist.

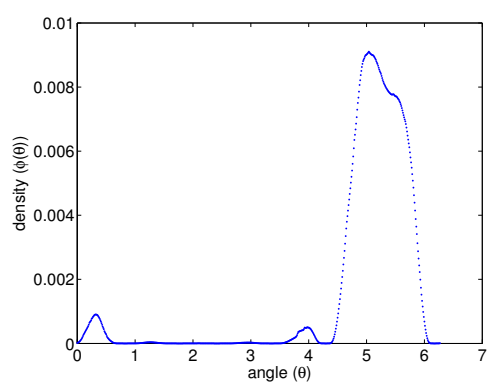

(b) With neighbor info., Exp dist.

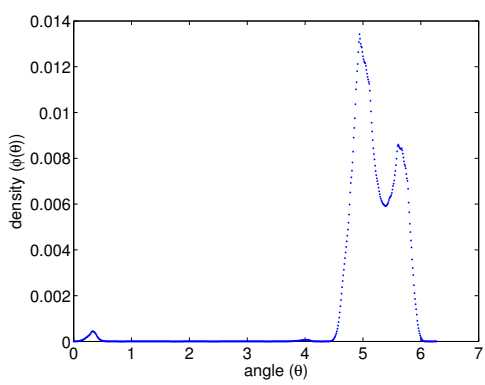

(c) With neighbor info., Gamma dist.

Fig. 7. Density function $\phi(\theta)$ from three methods.

and the resulted mean error is $354.4 \mathrm{~m}$ for $1 d B, 354.5 \mathrm{~m}$ for $2 d B$, and $354.6 \mathrm{~m}$ for $3 d B$. They are all slightly better than the fixed threshold approach but the improvements are insignificant in practice. The reason is that the reported SINRs are "good" SINR values. If we were given a "bad" SINR value, it would make a difference. Please refer to the examples in Fig. 2 which shows that a measured $\hat{\gamma}=0.5$ gives the similar distribution as $\gamma=1$ while the distribution based on $\hat{\gamma}=0.2$ is dramatically different.

\section{Neighbor information}

We show a real example on how incorporating the information that neighboring sectors have their SINR $\leq \nu$ would change the estimation. The mobile is attached to a sector with azimuth 5.23. We assume $\nu=0.05$ based on the observation in Section IV-B Without neighboring SINR considered, the density function is shown in Fig. 7(a) With neighboring SINR considered, the density function is shown in Fig. 7(b) Clearly with the influence of neighbor cells, the density function changes around 5.5 (to the right of the azimuth) and the angle of maximum density changes from 5.2 (Fig. 7(a)) to 5.0 (Fig. 7(b)).

The localization error does not get affected much, though, by taking into account the neighbor SINR information. This may be due to the fact that for most single-legged calls, the distance between the mobile and the associated base station is so small that the distance between the mobile and any neighboring base station will be large enough for the SINR from the neighboring base station to be lower than $\nu$. On the other hand, in some cases, the condition that SINR from the associated base station being greater than $\gamma$ implies the SINR from a neighboring base station is less than $\nu$, therefore the $g$ function in Eqn. (11) is dominated by the first term. Eqn. (10) is equivalent to Eqn. (3) in both cases.

\section{The distribution for fading}

We know from Section IV-A that the power attenuation factor can be modeled as a random variable that follows a Gamma distribution with mean 1 and shape factor between 1 and 2 . Therefore, instead of using exponential distribution, we can also model the power attenuation using a Gamma distribution with parameters $(2,0.5)$ wherein 2 is the shape parameter and 0.5 is the scale parameter. This random variable has mean $2 \times 0.5=1$ and the CCDF and PDF of this distribution are both quite simple: the PDF is $4 x e^{-2 x}$ and the CCDF is $(1+2 x) e^{-2 x}$. We plug in both the CCDF and PDF into Eqn. (10). We don't have a closed-from solution from the Gamma distribution for $\phi(\theta)$ but we can numerically calculate it. We consider the example above. Changing from exponential distribution to Gamma distribution again modifies the density function into Fig. 7(c) in which the density around 5.5 is further lowered.

When we apply Gamma distribution with shape $a=2$, scale $b=0.5$ to the estimation, the mean error increased to $360 \mathrm{~m}$. We guess it may be due to the fact that fading in the area under study, which is a suburban area, can be modeled better with an exponential distribution (or Gamma distribution with $a=1$ ) rather than the particular Gamma distribution with $a=2$. In other words, the "true" shape parameter of the Gamma distribution is closer to 1 than 2. However, if we were dealing with a more urban environment, the Gamma distribution with $a=2$ may work better. Repeating the evaluation with a different $a$ value (e.g., 1.5) and obtaining a data set from an urban area to compare the two distributions are part of our ongoing work.

\section{E. With RTT error}

In the previous subsections, we assumed that we have an accurate measurement of RTT. Section IV-C shows that the distance calculated from RTT measurement has an error that follows a Gaussian distribution with mean and standard deviation both around $0.3 \mathrm{~km}$. We take this distribution into Eqn. (12). The resulted mean localization error is around $399 m$, which is $20 \%$ lower comparing to the mean error of $501 \mathrm{~m}$ generated by the baseline approach.

\section{F. Discussion}

The authors in [8] theoretically calculated the mean localization error in UMTS networks to be $436 \mathrm{~m}$ for cells with three sectors, each with beamwidth $65^{\circ}$. They considered RTT measurements with $5 \mathrm{~m}$ precision, which is much more accurate than ours, and users assumed uniformly distributed inside each sector. This accuracy is close to what our baseline approach produces when the actual RTT is known.

There are various factors that impact the performance of our localization method. For example, a cell in the city may be a candidate for a fading model with Gamma distributed power attenuation rather than Rayleigh. $\beta=3$ may work better than $\beta=4$ in less populated areas while $\beta=5$ may work better in densely populated city centers. Another important factor is the a priori user distribution in a cell or sector. The assumption of uniform distribution may be invalid in some cells and the estimation based on a uniform distribution hence could be inaccurate. As a matter of fact, our data set comes with an artifact that a major fraction of E911 calls occurred at locations 
near a freeway, and a major fraction of cells are located next to a freeway and as a result of network engineering, each of these cells have at least one sector roughly aligned with the freeway on their azimuth. For these sectors, the a priori user distribution would be highly non-uniform with a higher density along the direction of the freeway. To demonstrate this, we show two sectors where the Bayesian method perform poorly in Figs. 8 (a) and (b), because the two sectors are aligned with a major road or a freeway. As contrast, two sectors where the method performs well are a residential area sector (Fig. 8.(c)) and one facing a freeway (Fig. 8 (d)). A better knowledge of user's a priori distribution in a cell based on the cell's geographical location would improve the localization accuracy.

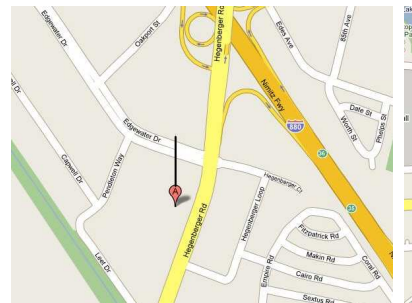

(a) Bad sector 1

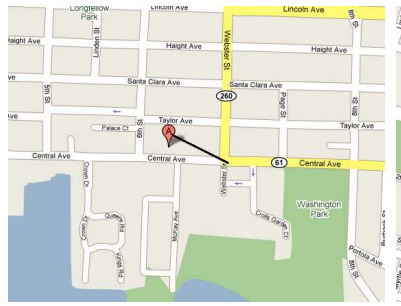

(c) Good sector 1

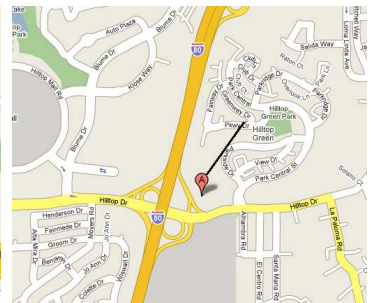

(b) Bad sector 2

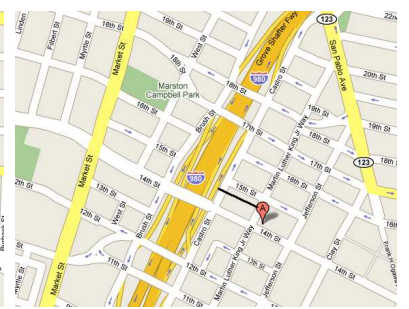

(d) Good sector 2
Fig. 8. Examples of sectors where the Bayesian method does not perform well and does perform well. Black lines indicate sector azimuth.

\section{CONCLUSION}

Motivated by transforming large-scale CDRs to location records, we solved the localization problem when triangulation and/or trilateration cannot be performed due to lack of measurement of more than one base station. Our goal is to localize a mobile with only information of the associated base station. We developed a Bayesian-based method which calculates the density around the associated base station based on information such as path-loss, the SINR threshold for a user to be associated or not to be associated with a base station, and the network layout. Then users were localized via the maximum likelihood approach, i.e., as where the density is maximum. We performed measurements in a commercial network to "tune" the parameters used in this method, including the SINR thresholds, the interference from non-neighboring base stations, the RTT measurement error, etc. We then validated this method using three months of E911 records in a switch area of a CDMA2000 network. We demonstrated that by incorporating the aforementioned information, we reduced the mean localization error by about $20 \%$ compared to a blind approach which places users uniformly in a sector along the arc that is at a most probable distance to the base station based on the RTT measurement.

The Bayesian method is proved to be sensitive to parameter settings such as $\beta$ which governs the power attenuation. Our results indicate that the accuracy of the method can be improved with a better knowledge of a priori user distribution. As future work, we plan to develop a localization framework to deal with heterogeneous network environments: we profile cells first with regard to whether they are in the city, suburban or rural areas, and refine their a priori user distribution through the neighborhood information. Then when localization is performed, the fading distribution and parameters and a priori user distribution are selected to match each cell's profile. For example, for a suburban cell, we will use $\beta=4$ and the Rayleigh fading; for an urban cell, we will assume the power attenuation follows a Gamma distribution with the parameter stored in the profile; for a rural cell, we will use $\beta=3$. We will assume a non-uniform a priori user distribution in cells where a highway traverses.

\section{REFERENCES}

[1] International Telecommunication Union, Measuring the Information Society: The ICT Development Index, 2009.

[2] J.-P. Onnela et al, "Structure and Tie Strengths in Mobile Communication Networks," New Journal of Physics, vol. 9, 2007.

[3] G. Palla, A.-L. Barabasi, and T. Vicsek, "Quantifying social group evolution," Nature, vol. 446, no. 664, 2007.

[4] D. Borckmann, L. Hufnagel, and T. Geisel, "The scaling laws of human travel," Nature, vol. 439, pp. 462-465, 2006.

[5] L. Backstrom, C. Dwork, and J. Kleinberg, "Wherefore art thou r3579x?: anonymized social networks, hidden patterns, and structural steganography," in Proc. the 16th international conference on World Wide Web, Banff, Alberta, Canada, 2007, pp. 181-190.

[6] N. Bulusu, J. Heidemann, and D. Estrin, "GPS-less low cost outdoor localization for very small devices," IEEE Personal Communications Magazine, vol. 7, no. 5, pp. 28-34, October 2000.

[7] S. Y. Willassen, "A method for implementing mobile station location in GSM," Master's Thesis, Norwegian University of Science and Technology, 1998

[8] J. Borkowski, J. Niemel, and J. Lempiinen, "Performance of cell ID+RTT hybrid positioning method for UMTS radio networks," in Proc. 5th European Wireless Conference, 2004.

[9] J. J. Caffery and G. Stüber, "Overview of radiolocation in CDMA cellular systems," IEEE Communications Magazine, April 1998.

[10] V. Otsason, A. Varshavsky, A. LaMarca, and E. de Lara, "Accurate GSM indoor localization," in Proc. UbiComp, 2005.

[11] A. Kupper, Location-based Services: Fundamentals and Operation. John Wiley \& Sons, 2005.

[12] J. H. Reed, K. J. Krizman, B. D. Woerner, and T. S. Rappaport, "An overview of the challenges and progress in meeting the E-911 requirement for location service," IEEE Communications Magazine, 1998.

[13] H. Tang, Y. Park, and T. Qiu, "A TOA-AOA-based NLOS error mitigation method for location estimation," EURASIP J. Adv. Signal Process, vol. 8, no. 1 , pp. $1-14,2008$.

[14] Z. Gu and E. Gunawan, "Radiolocation in CDMA cellular system based on joint angle and delay estimation," Wirel. Pers. Commun., vol. 23, no. 3, pp. 297-309, 2002.

[15] M. Sortais, S. D. Hermann, and A. Wolisz, "Analytical investigation of intersection based range-free localization," in Proc. European Wireless, 1997.

[16] M. Y. Chen and et al., "Practical metropolitan-scale positioning for GSM phones," in Proc. UbiComp, 2006.

[17] D. Fox, J. Hightower, L. Liao, D. Schulz, and G. Borriello, "Bayesian filtering for location estimation," IEEE Pervasive Computing magazine, vol. 2, no. 3, pp. 24-33, July 2003.

[18] A. M. Ladd and et al., "On the feasibility of using wireless ethernet for indoor localization," IEEE Transactions on Robotics and Automation, vol. 20, no. 3, pp. 555-9, June 2004

[19] A. LaMarca and et al., "Place lab: Device positioning using radio beacons in the wild," in Proc. Pervasive Computing, 2005.

[20] A. Okabe, B. Boots, K. Sugihara, and S. N. Chiu, Spatial Tessellations. Wiley, 1992

[21] D. Tse and P. Viswanath, Fundamentals of Wireless Communication. Cambridge University Press, 2005.

[22] F. Baccelli and B. Błaszczyszyn, "On a coverage process ranging from the Boolean model to the Poisson Voronoi tessellation, with applications to wireless communications," Adv. Appl. Probab., vol. 33, 2001.

[23] B. Blaszczyszyn and M. A. Tran, "Three-sector-antenna path-loss and CDMA downlink capacity," Rapport de stage d'option, Ecole Polytechnique, France, September 2003.

[24] "Agilent W1314A Multi-band Wireless Measurement Receiver Data Sheet," http://cp.literature.agilent.com/litweb/pdf/5989-7970EN.pdf.

[25] "Thermal Noise Calculator." http://www.vk2zay.net/calculators/thermalnoise.php. 